\title{
Análise Acústica de Fachadas de Light Steel Frame e Placas Cimentícias
}

\section{Acoustic analysis of Facades of Light Steel Framing and Flat Sheets}

\author{
Thais Sacomani Zenerato ${ }^{1}$, Juliana Furtado Arrobas Martins ${ }^{2}$, \\ Stelamaris Rolla Bertoli ${ }^{2}$, Carlos Eduardo Marmorato Gomes ${ }^{2}$, \\ Patricia Stella Pucharelli Fontanini ${ }^{2}$
}

\footnotetext{
${ }^{1}$ Faculdade de Engenharia Civil, Arquitetura e Urbanismo - Universidade Estadual de Campinas - UNICAMP - Rua Saturnino de Brito, 224, CEP: 13.083-889, Campinas, São Paulo, Brasil.

${ }^{2}$ Faculdade de Engenharia Civil, Arquitetura e Urbanismo - UNICAMP, Campinas, São Paulo, Brasil. e-mail: thais.zenerato@hotmail.com, juarrobas@hotmail.com,rolla@fec.unicamp.br, cemgomes@fec.unicamp.br, patricia@fec.unicamp.br
}

\section{RESUMO}

A construção a seco tem sido considerada uma alternativa tecnológica aos sistemas construtivos convencionais e, no Brasil, sistemas como o Light Steel Frame e Drywall passaram a ser empregados em maior escala nos últimos anos. Basicamente, estes sistemas consistem em painéis estruturados por perfis de aço, preenchidos internamente por uma lã de isolamento e fechados com placas planas nas faces externa e interna. Foi verificado que, no Brasil, as placas mais usadas no fechamento externo destes painéis são produzidas à base de cimento Portland e fibras, sendo, então, comumente denominadas de fibrocimento. Contrapondo, percebe-se na China e EUA alternativas como placas fabricadas com óxido de magnésio, denominada placas planas magnesianas, cuja aplicação tem sido incipiente pela construção brasileira. A norma de desempenho para habitações residenciais (NBR 15.575), propõe uma análise acústica destes painéis a partir de um protótipo, adotando como variável a placa plana de fechamento. Foram então estudadas as diferenças no isolamento de sons aéreos avaliado a partir da medição do parâmetro $\mathrm{D}_{2 \mathrm{~m}, \mathrm{nT}}$ em função de frequência segundo a norma ISO 140-5 e o cálculo do parâmetro $\mathrm{D}_{2 \mathrm{~m}, \mathrm{nT} \text {, w }}$ (ISO 717-1), tendo como critério a norma NBR15575-4. Os resultados apresentados neste trabalho demonstraram que as placas planas à base de magnésio podem colaborar para um melhor isolamento sonoro em toda faixa de frequência, sendo observado um ganho de $4 \mathrm{~dB}$ no isolamento comparativamente às placas convencionais de fibrocimento.

Palavras-chave: Placa de fibrocimento. Cimento magnesiano. Desempenho acústico. Construção a seco.

\section{ABSTRACT}

Dry construction has been considered as a technological alternative to conventional construction systems, and in recent years, systems as Light Steel Frame and Drywall have been used on a larger scale in Brazil. These systems consist of structured panels by steel profiles, filled internally by an insulation wool and sealed with flat plates on internal and external faces. It was verified that, in Brazil, the most used slabs in the external closure of these panels are produced from Portland cement and fibers, and there are therefore commonly referred how fiber cement. In the China and the US, they have alternatives such as plates made with magnesium oxide, called flat magnesium plates, whose application has been incipient by the Brazilian work construction. The performance standardization for popular residential (NBR 15.575), it was proposed an acoustic analysis of these panels from a special prototype, adopting as variable the plane flat. The differences in air sound insulation evaluated from the measurement of the parameter D2m, nT as a function of frequency according to ISO 140-5 and the calculation of the parameter D2m, nT, w (ISO 717-1) were studied, having as criterion the NBR 15575-4. The results presented in this work demonstrated that the magnesium based flat plates can collaborate for a better sound insulation throughout the frequency range, with a gain of $4 \mathrm{~dB}$ in the insulation compared to conventional fiber cement panels.

Keywords: Fiber cement panels. Magnesium cement, Acoustic performance, Dry construction. 


\section{INTRODUÇÃO}

Desde seu surgimento por volta do ano de 1820, o cimento Portland tem sido o aglomerante mais utilizado na construção civil, porém, sua produção está relacionada a grandes emissões de gás carbônico. A partir do relatório da CNI [1], grande parte do $\mathrm{CO}_{2}$ gerado na produção deste material é proveniente da descarbonatação do calcário (50\%), combustão no forno (30\%), transporte de matérias-primas (5\%) e consumo elétrico (5\%). Em relação aos cimentos comerciais, a média brasileira pode ser avaliada em torno de 650 quilos de $\mathrm{CO}_{2}$ por tonelada produzida, conforme Confederação Nacional da Indústria (CNI, 2012). Estes dados fazem a indústria cimenteira mundial ser responsável por aproximadamente $3 \%$ das emissões de gases causadores do efeito estufa e por aproximadamente 5\% das emissões de $\mathrm{CO}_{2}$, conforme HUMPHREYS e MAHA MAHASENAN [2].

Por sua vez, a descoberta do cimento à base de óxido de magnésio ocorreu em 1867, e compósitos magnesianos têm sido usados como aglomerantes em placas e painéis, materiais isolantes e em várias outras aplicações. (THOMAS) [3]. Atualmente, no Brasil, grande parte das jazidas e estoques de matéria-prima para produção do óxido de magnésio estão em Brumado, cidade do Estado da Bahia (BA), porém, seu uso tem sido restrito à produção de materiais refratários.

Contrapondo, a fabricação de componentes construtivos à base de óxido de magnésio $(\mathrm{MgO})$ tem sido crescente em países tais como os Estados Unidos e Austrália, apesar de estar ainda concentrada na Ásia, especialmente na China, aonde estes elementos são largamente empregados em habitações populares. A primeira aplicação em larga escala desse cimento ocorreu há mais de 10 anos, motivada pelos Jogos Olímpicos de 2008, utilizando placas de $\mathrm{MgO}$ para tornar a cidade um modelo de construção ecológica e desenvolvimento sustentável (BEIJING REPORT) [4]. A segunda grande aplicação do $\mathrm{MgO}$ foi no edifício LEED Platinum, em Taiwan, que contém placas de MgO nas paredes e tetos (TAMBOLI et al) [5].

Destaca-se a utilização de aglomerantes menos agressivos ao meio ambiente têm sido uma preocupação constante no setor da construção civil. O cimento magnesiano, também denominado como cimento Sorel, pode ser produzido através de uma reação química entre o $\mathrm{MgO}$ (óxido de magnésio) e um sal de caráter ácido, como cloreto de magnésio, fosfato de amônio ou sulfato de magnésio. Respectivamente, estes compostos são denominados MOC (oxi-cloreto de magnésio), MPC (fosfato de magnésio) e MOS (oxisulfato de magnésio).

Apesar do Brasil ser um dos maiores produtores do $\mathrm{MgO}$, a produção nacional de placas planas é realizada por meio de um processo denominado Hatschek, desenhado especificamente para uso do cimento Portland. Neste sentido, em virtude da escassez de placas magnesianas no mercado nacional, neste trabalho utilizou-se placas chinesas produzidas por meio da tecnologia à base de oxi-cloreto de magnésio. Conforme SHAND [6], estes compostos possuem propriedades mecânicas muitas vezes superiores às do cimento Portland, bem como apresentam bom comportamento ao fogo e baixa condutividade térmica. Sendo assim, podem ser suscetíveis em ambientes saturados. Neste sentido, acredita-se que as fases mais estáveis destes compostos são as fases denominadas (3-1-8) - $\{3 \mathrm{Mg}(\mathrm{OH}) 2 \cdot \mathrm{MgCl} 2.8 \mathrm{H} 2 \mathrm{O}\}$ e, (5-1-8) $\{5 \mathrm{Mg}(\mathrm{OH}) 2 . \mathrm{MgCl} 2.8 \mathrm{H} 2 \mathrm{O}\}$ e $\{\mathrm{Mg}(\mathrm{OH}) 2\}$, que apresentam maior durabilidade.

Dentre os elementos construtivos, as placas planas produzidas com óxido de magnésio se destacam especialmente por sua utilização de processos construtivos como o Light Steel Frame (LSF), uma concepção construtiva que se caracteriza por perfis metálicos parafusados, aonde são afixadas as placas em suas faces externa e interna. Neste sentido, o Grupo de Estudos em Materiais de Construção, Compósitos e Cimentícios (GMC3), da Faculdade de Engenharia Civil, Arquitetura e Urbanismo da UNICAMP, tem sido pioneiro na promoção desta tecnologia.

A proposta de verificação de sua aplicabilidade foi então baseada nos requisitos básicos relacionados ao conforto acústico das construções habitacionais, o que implica na verificação do cumprimento da norma de desempenho NBR 15575 [4]. A relevância desta análise é destacada pela World Health Organization, que demonstra que a exposição ao ruído causa uma série de mudanças no comportamento e nos costumes sociais dos moradores de residências (WHO) [7].

Dessa forma, o desempenho acústico é um item de cumprimento obrigatório da NBR 15575 [8], independentemente do sistema construtivo utilizado. Neste item, são especificados os requisitos mínimos que devem ser atendidos e prescrições de níveis de desempenho, indicando qual o isolamento requerido em relação ao sistema de vedações verticais internas e externas. Para fachadas, o parâmetro acústico que indica o índice do isolamento sonoro aéreo é a Diferença Padronizada de Nível Ponderada (D2m,nT,w ) e seu valor normativo varia de acordo com a localização da edificação. Os valores exigidos para isolamento de fachadas estão apresentados na Tabela 1. 
Tabela 1: Diferença Padronizada de Nível Ponderada da vedação externa, $\mathrm{D}_{2 \mathrm{~m}, \mathrm{nT}, \mathrm{w}}$, para ensaios em campo

\begin{tabular}{|c|c|c|c|}
\hline $\begin{array}{l}\text { Classe de } \\
\text { Ruído }\end{array}$ & Localização da habitação & $\begin{array}{l}\mathrm{D} 2 \mathrm{~m}, \mathrm{nT}, \mathrm{w} \\
\quad(\mathrm{dB})\end{array}$ & $\begin{array}{c}\text { Nível de } \\
\text { desempenho }\end{array}$ \\
\hline \multirow{3}{*}{1} & \multirow{3}{*}{$\begin{array}{l}\text { Habitação localizada distante de fontes de ruído intenso de } \\
\text { quaisquer naturezas }\end{array}$} & $\geq 20$ & M \\
\hline & & $\geq 25$ & I \\
\hline & & $\geq 30$ & $S$ \\
\hline \multirow{3}{*}{11} & \multirow{3}{*}{$\begin{array}{l}\text { Habitação localizada em áreas sujeitas a situações de ruído } \\
\text { não enquadráveis nas classes } 1 \text { e } 11 .\end{array}$} & $\geq 25$ & M \\
\hline & & $\geq 30$ & I \\
\hline & & $\geq 35$ & S \\
\hline \multirow{3}{*}{111} & \multirow{3}{*}{$\begin{array}{l}\text { Habitação sujeita a ruído intenso de meios de transporte e de } \\
\text { outras naturezas, desde que conforme a legislação; }\end{array}$} & $\geq 30$ & M \\
\hline & & $\geq 35$ & I \\
\hline & & $\geq 40$ & S \\
\hline
\end{tabular}

Fonte: NBR 15575-4 (2013)

Desta forma, com o propósito de verificar a influência das placas de fechamento no desempenho acústico dos painéis de Light Steel Frame, foram estudadas especialmente as diferenças no isolamento de sons aéreos tendo como critério a norma NBR15575-4. Os resultados demonstraram que as placas planas à base de magnésio podem colaborar para um melhor isolamento sonoro em toda faixa de frequência, sendo observado um ganho de $4 \mathrm{~dB}$ no isolamento comparativamente às placas convencionais de fibrocimento.

\section{MATERIAIS E MÉTODOS}

O trabalho foi desenvolvido utilizando um protótipo experimental de Light Steel Frame e dois módulos construtivos apresentando dimensões de $2,5 \mathrm{~m}$ x $2,5 \mathrm{~m}$ em planta baixa e, altura de 3,0m. Também foram empregados perfis de aço fabricados a frio (montantes e guias) com espessura $0,95 \mathrm{~mm}$ e espaçamentos entre as montantes de $0,40 \mathrm{~m}$. A estrutura teve seu contraventamento realizado por meio de placas OSB (Oriented strand board) com dimensões de $1,20 \mathrm{~m}$ x 2,40m, espessura de $11 \mathrm{~mm}$ e densidade de $658 \mathrm{~kg} / \mathrm{m}^{3}$. Foram usadas placas cimentícias convencionais na parte interna, adotando-se como variável apenas o fechamento externo.

Na tabela 2 são apresentadas as propriedades físicas das placas empregadas.

Tabela 2: Propriedades físicas das placas planas utilizadas na pesquisa.

\begin{tabular}{c|c|c|c|c|c}
\hline \multicolumn{3}{c|}{ Placa Plana Magnesiana } & \multicolumn{3}{c}{ Placa Plana de Fibrocimento } \\
\hline $\begin{array}{c}\text { Absorção } \\
(\%)\end{array}$ & $\begin{array}{c}\text { Índice de } \\
\text { Vazios } \\
(\%)\end{array}$ & $\begin{array}{c}\text { Densidade } \\
\left(\mathrm{g} / \mathrm{cm}^{3}\right)\end{array}$ & Absorção $(\%)$ & $\begin{array}{c}\text { Índice de } \\
\text { Vazios } \\
(\%)\end{array}$ & $\begin{array}{c}\text { Densidade } \\
\left(\mathrm{g} / \mathrm{cm}^{3}\right)\end{array}$ \\
\hline 35,2 & 33,0 & 0,94 & 16,6 & 28,4 & 1,72 \\
\hline
\end{tabular}

O espaço interno entre placas OSB foi preenchido com lã de vidro com densidade de $43 \mathrm{~kg} / \mathrm{m}^{3}$. O forro foi executado com placas de gesso acartonado com espessura de $12,5 \mathrm{~mm}$ e a cobertura em duas águas de $45^{\circ}$ e telhas de fibrocimento sem amianto de espessura de $5 \mathrm{~mm}$.

A figura 1 apresenta as principais etapas construtivas do protótipo. 

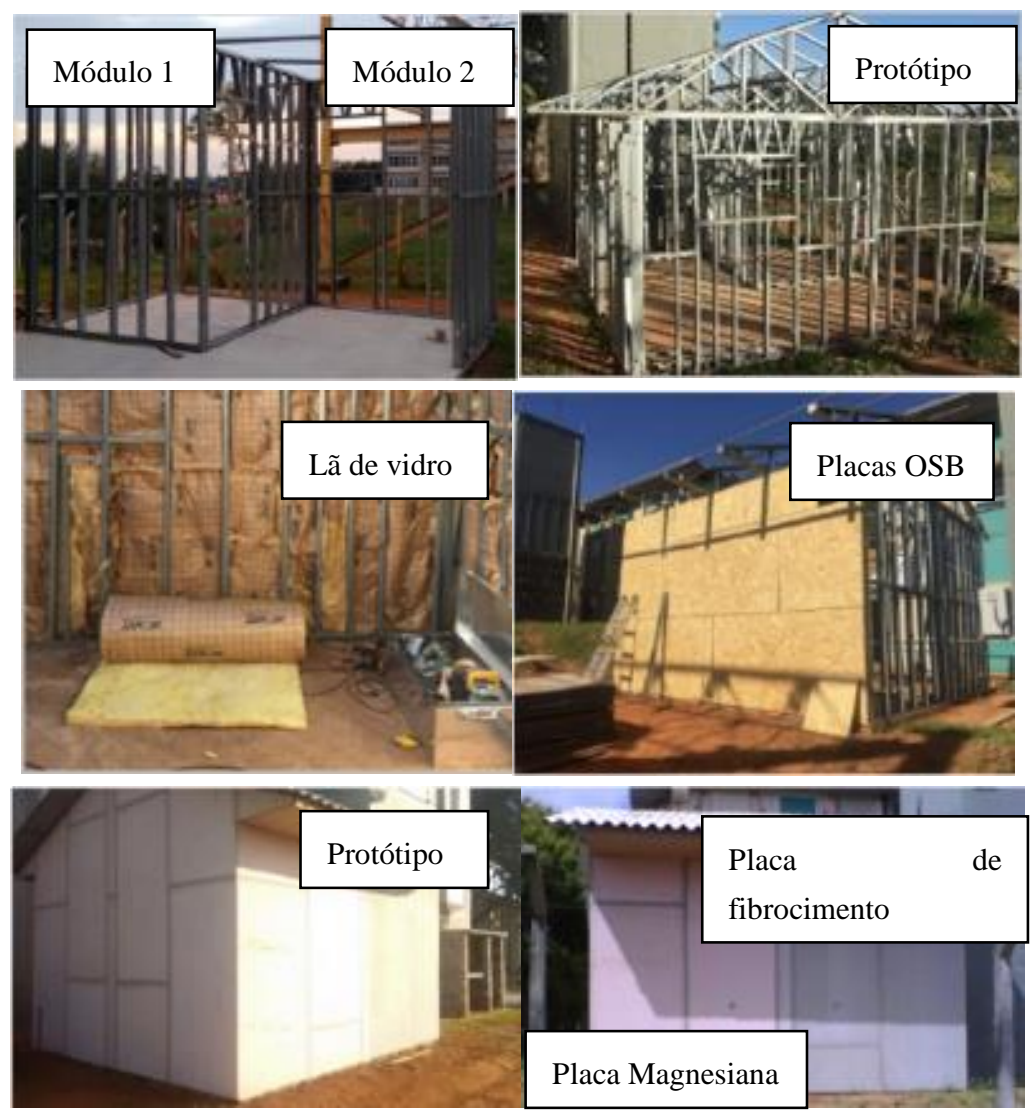

Figura 1: Etapas construtivas do protótipo do Light Steel Frame.

Na Figura 2 observa-se os detalhes construtivos bem como a fixação das placas de fibrocimento (Painel/corte A) e cimento magnesiano (Painel/corte B), em conjunto com as placas de OSB para contraventamento e a lã de vidro para isolamento. O painel de vedação foi então formado pela sequência de placa cimentícia, placa OSB e lã de vidro, repetindo a placa de OSB e, novamente, finalizando com a placa cimentícia pelo lado interno. A espessura total desse conjunto então, foi formada por $10 \mathrm{~mm}$ de cada placa cimentícia, $11 \mathrm{~mm}$ de cada placa de OSB, 95mm dos perfis de aço, com a lã de vidro (20mm) no interior, totalizando uma parede de aproximadamente $140 \mathrm{~mm}$.

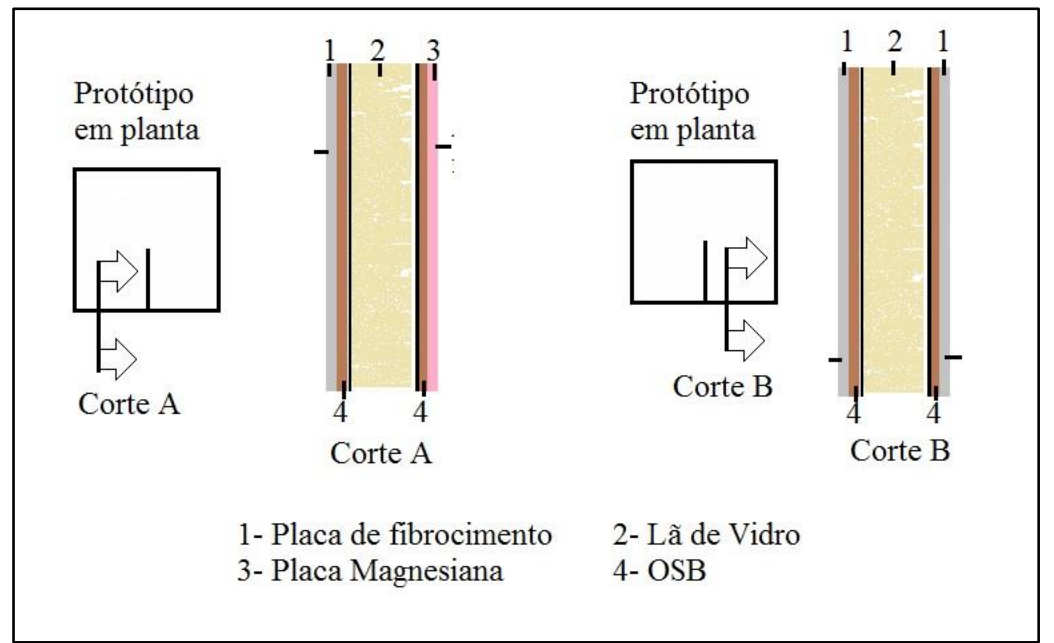

Figura 2: Planta e cortes em escala - (a) Painel com placa magnesiana - (b) Painel com placa de fibrocimento.

Como mencionado, a norma NBR 15575-4 [8], a mesma apresenta requisitos, métodos e critérios para a determinação do desempenho de isolamento sonoro aéreo de sistemas de vedações verticais externas e internas das edificações de interesse social. O critério para o desempenho é respaldado em valores do 
parâmetro acústico Diferença Padronizada de Nível Ponderada (D2m,nT,w) determinado segundo a norma ISO 717-1 [9] a partir dos valores calculados da Diferença Padronizada de Nível (D2m,nT) obtidos em função de frequência. O método indicado pela NBR 15575-4 [8] para a determinação do isolamento a sons aéreos de partições verticais externas é o procedimento proposto pela norma ISO 140-5 [10]. Este procedimento de medição é independentemente do tipo de fachada ou do tipo de edificação a ser avaliada, ele foi escolhido como o método para determinar o isolamento sonoro aéreo das placas de cimento aplicadas como fachada de um protótipo construído em Light Steel Frame (LSF). Para efeito de comparação foram avaliadas o desempenho acústico de placas de cimento preparadas com cimento a base de óxido de magnésio e a base de cimento Portland.

Do lado externo da edificação foi posicionada uma fonte sonora e emitindo ruído rosa, o procedimento de medição estabelece que sejam obtidos os níveis de pressão sonora a $2 \mathrm{~m}$ da fachada (L1) e os níveis de pressão sonora no ambiente interno da fachada estudada (L2), ambos medidos em função de frequência em bandas de $1 / 3$ de oitava entre as frequências de $100 \mathrm{~Hz}$ e $3150 \mathrm{~Hz}$. No ambiente interno, denominado de sala receptora, também se mede o tempo de reverberação $(\mathrm{T})$ em função de frequência em bandas de 1/3 de oitava no mesmo intervalo de frequência. Com base nos valores de (L1), (L2) e (T) calcula-se a Diferença Padronizada de Nível (D2m,nT) em função de frequência empregando a equação 1:

$\mathrm{D}_{2 \mathrm{~m}, \mathrm{nT}}=\mathrm{L}_{1,2 \mathrm{~m}}-\mathrm{L} 2+10 \log (\mathrm{T} / \mathrm{To})$

em que L1,2m é o nível de pressão sonora medido a $2 \mathrm{~m}$ da fachada, (L2) é o nível de pressão sonora no ambiente interno da fachada estudada, T é o tempo de reverberação do ambiente interno e To é tempo de reverberação de referência que vale $0,5 \mathrm{~s}$.

O sistema de medição foi composto pelo medidor de nível de pressão sonora BK 2270, a fonte omnidirecional Tipo 4292-L, o amplificador Tipo 2734 e programa Building Acoustics BZ 7228, todos da Bruel\&Kjaer.

Externamente, o posicionamento da fonte sonora obedeceu às distâncias recomendadas pela norma ISO 140-5 (1998). Na Figura 3 são apresentadas a posição e distância da fonte sonora em relação a fachada finalizada com a placa magnesiana (FM1) e com a placa de fibrocimento Portland (FP1).

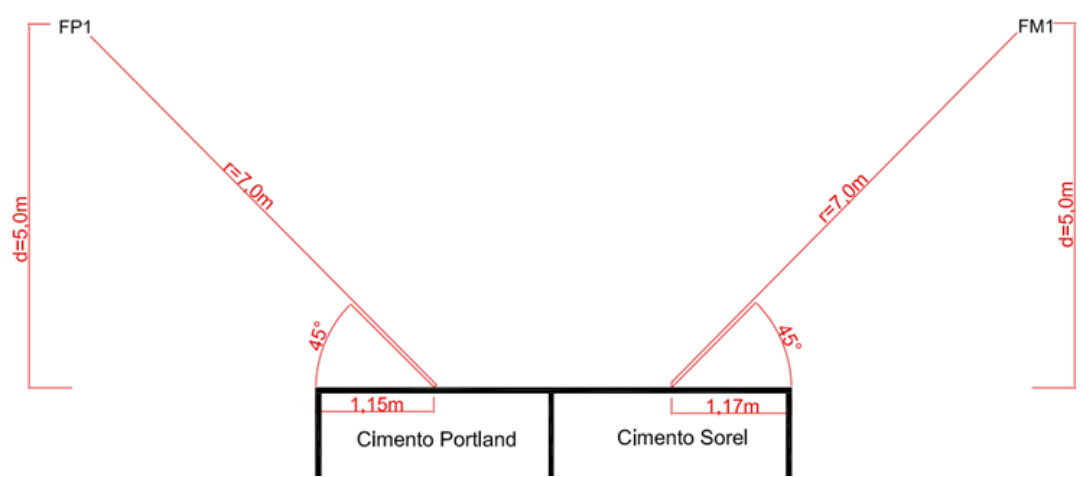

Figura 3: Representação, em planta, do posicionamento da fonte sonora na área externa.

Para as medidas dos níveis de pressão sonoro externos (L1) foram escolhidas três posições de microfones em frente a fachada com placa Sorel (M1, M2 e M3) e três posições em frente a fachada com a placa de fibrocimento Portland (P1, P2 e P3). Todos os pontos de microfone estão distantes $2 \mathrm{~m}$ do plano da fachada e a uma altura $\mathrm{h}=1,5 \mathrm{~m}$. A Figura 4 indica as posições escolhidas. 


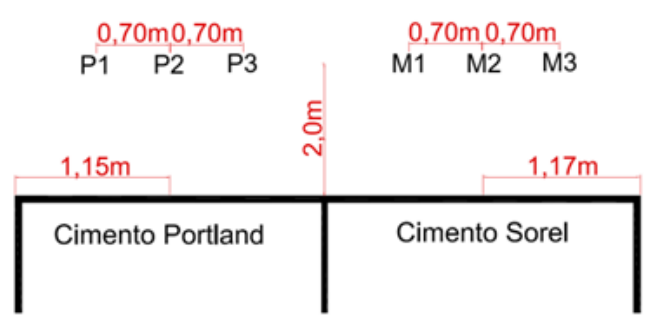

Figura 4: Representação, em planta, do posicionamento dos microfones na área externa.

Para as medições dos níveis de pressão sonora na parte interna da edificação (L2), de acordo com a norma ISO 140-5 devem ser utilizadas na sala receptora um mínimo de cinco posições de microfone, distribuídos com o maior espaçamento possível. A norma também estabelece uma distância mínima de $0,7 \mathrm{~m}$ entre posições de microfone e de $0,5 \mathrm{~m}$ entre a posição do microfone e das bordas do ambiente. Devido as dimensões reduzidas dos ambientes do protótipo, foram escolhidas três posições de microfone em cada ambiente e utilizadas duas alturas diferentes, $\mathrm{h} 1=1,45 \mathrm{~m}$ e $\mathrm{h} 2=1,73 \mathrm{~m}$. Totalizando seis medições (M4, M5 e M6) para a sala receptora referente a placa magnesiana e outras seis medições (P4, P5 e P6) referente a placa de fibrocimento Portland. As posições são representadas na Figura 5.

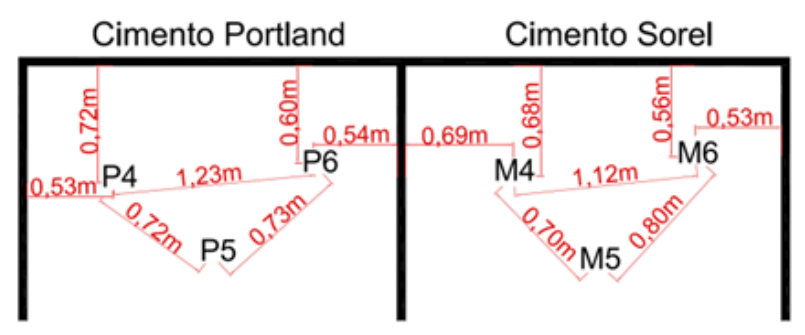

Figura 5: Representação, em planta, do posicionamento dos microfones na área interna.

Para a obtenção do tempo de reverberação (T), a fonte sonora foi posicionada no interior da edificação a uma altura de 1,5m. A norma ISO 140-5 estabelece uma distância mínima de 1,0m entre a fonte e quaisquer posições de microfone. A Figura 5 mostra o posicionamento da fonte sonora e dos pontos de medição mais próximos à fonte, no interior da edificação para a medição do tempo de reverberação $(\mathrm{T})$.

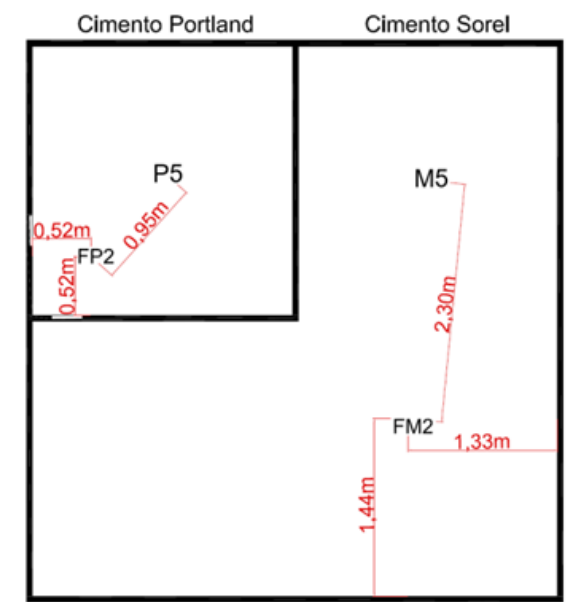

Figura 6: Representação, em planta, do posicionamento da fonte sonora na área interna.

O nível de pressão sonora relativo ao ruído de fundo foi medido no interior da sala receptora para verificar a necessidade ou não da correção do nível L2 pelo ruído de fundo conforme recomenda a ISO 1405. A correção não é necessária quando a diferença entre o ruído de fundo e o nível sonoro com a fonte ligada 
externamente é maior que $10 \mathrm{~dB}$. Nesse ensaio não foi necessário efeituar a correção. Considerando os resultados medidos de L1, L2 e T em função de frequência, e empregando a Equação 1 são calculados os valores do parâmetro Diferença Padronizada de Nível (D2m,nT) obtidos em função de frequência. A norma ISO 717-1 apresenta o procedimento para que os valores da Diferença Padronizada de Nível, obtidos em função de frequência, sejam convertidos em um único número e que irá representar o desempenho acústico da fachada.

Na norma ISO 717-1 são disponibilizados os valores de uma curva de referência, que estão apresentados na Tabela 3. Foi plotado um gráfico com as Diferenças Padronizada de Nível obtidos e com os valores da curva disponibilizada. A curva foi ajustada com incrementos de $1 \mathrm{~dB}$, até que a soma dos desvios desfavoráveis fosse o maior possível, mas não superior a 32dB. Por desvios desfavoráveis é entendido a diferença entre os valores da Diferença Padronizada de Nível e a curva de referência, quando estes são inferiores aos valores da referida curva. A Diferença Padronizada de Nível Ponderada do sistema avaliado corresponde ao valor da curva de referência ajustada, na frequência de $500 \mathrm{~Hz}$.

Tabela 3: Valores da curva de referência para som aéreo - Fonte: adaptado da ISO 717-1 [5]

\begin{tabular}{c|c|c|c}
\hline Frequência & Valores de Referência & Frequência & Valores de Referência \\
\hline $\mathrm{Hz}$ & $\mathrm{dB}$ & $\mathrm{Hz}$ & $\mathrm{dB}$ \\
\hline 100 & 33 & 630 & 53 \\
\hline 125 & 36 & 800 & 54 \\
\hline 160 & 39 & 1000 & 55 \\
\hline 200 & 42 & 1250 & 56 \\
\hline 250 & 45 & 1600 & 56 \\
\hline 315 & 48 & 2000 & 56 \\
\hline 400 & 51 & 2500 & 56 \\
\hline 500 & 52 & 3150 & 56 \\
\hline
\end{tabular}

\section{RESULTADOS E DISCUSSÕES}

Os valores da Diferença Padronizada de Nível $\left(\mathrm{D}_{2 \mathrm{~m}, \mathrm{nT}}\right)$ em função da frequência em bandas de 1/3 de oitava entre $100 \mathrm{~Hz}$ e $3150 \mathrm{~Hz}$ foram calculados segundo a Equação 1, com base nos valores medidos de L1, 2m, L2 e $\mathrm{T}$ conforme procedimento descrito na metodologia. Os resultados de D2m,nT em função de frequência para as placas produzidas com cimento Portland e com cimento à base de óxido de magnésio são apresentados na Tabela 4.

Tabela 4: Diferença Padronizada de Nível em função de frequência das placas estudadas.

\begin{tabular}{c|c|c}
\hline \multirow{2}{*}{$\begin{array}{c}\text { Frequência } \\
(\mathbf{H z})\end{array}$} & \multicolumn{2}{|c}{$\mathbf{D}_{\mathbf{2 m , \mathbf { n } T}}(\mathbf{d B})$} \\
\cline { 2 - 3 } & Portland & Sorel \\
\hline 100 & 19,4 & 17,9 \\
\hline 125 & 20,3 & 24,6 \\
\hline 160 & 16,8 & 18,1 \\
\hline 200 & 18,3 & 22,3 \\
\hline 250 & 19,0 & 26,0 \\
\hline 315 & 21,9 & 20,7 \\
\hline 400 & 18,5 & 22,4 \\
\hline 500 & 21,8 & 25,7 \\
\hline 630 & 23,7 & 26,4 \\
\hline 800 & 24,5 & 24,5 \\
\hline
\end{tabular}




\begin{tabular}{l|l|l}
\hline 1000 & 13,8 & 24,1 \\
\hline 1250 & 18,8 & 27,1 \\
\hline 1600 & 22,5 & 28,5 \\
\hline 2000 & 20,3 & 26,1 \\
\hline 2500 & 24,6 & 26,0 \\
\hline 3150 & 23,5 & 28,3 \\
\hline
\end{tabular}

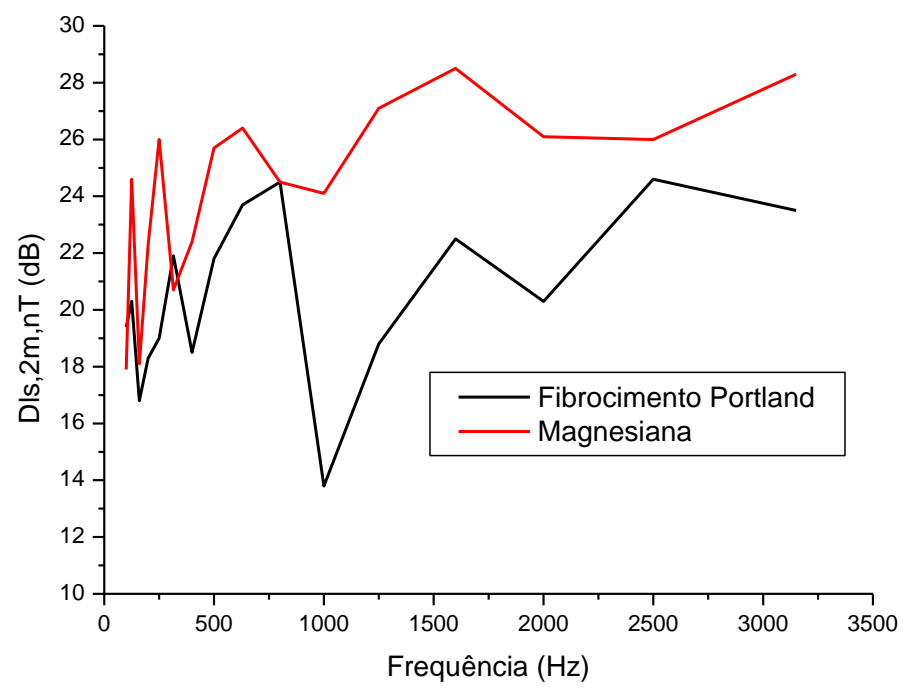

Figura 7: Diferença Padronizada de Nível das fachadas.

Para efeitos de comparação, a Figura 7 apresenta as curvas da Diferença Padronizada de Nível em função de frequência para as duas fachadas. Observa-se que a placa magnesiana tem isolamento superior à placa de fibrocimento Portland em toda a faixa de frequência, exceto para as frequências de $100 \mathrm{~Hz}$ e $315 \mathrm{~Hz}$. Destaca-se que a placa fibrocimento Portland tem uma redução acentuada no isolamento a $1000 \mathrm{~Hz}$, frequência importante para isolamento de sons relativos a fala. Esse decréscimo no isolamento nessa frequência está associado ao efeito de coincidência. A troca de cimento favoreceu o isolamento nessa frequência especifica.

Nas Figuras 8 e 9 estão as curvas de referência da norma ISO 717-1 [9] ajustadas para os resultados alcançados da Diferença Padronizada de Nível em função de frequência para as duas fachadas. 


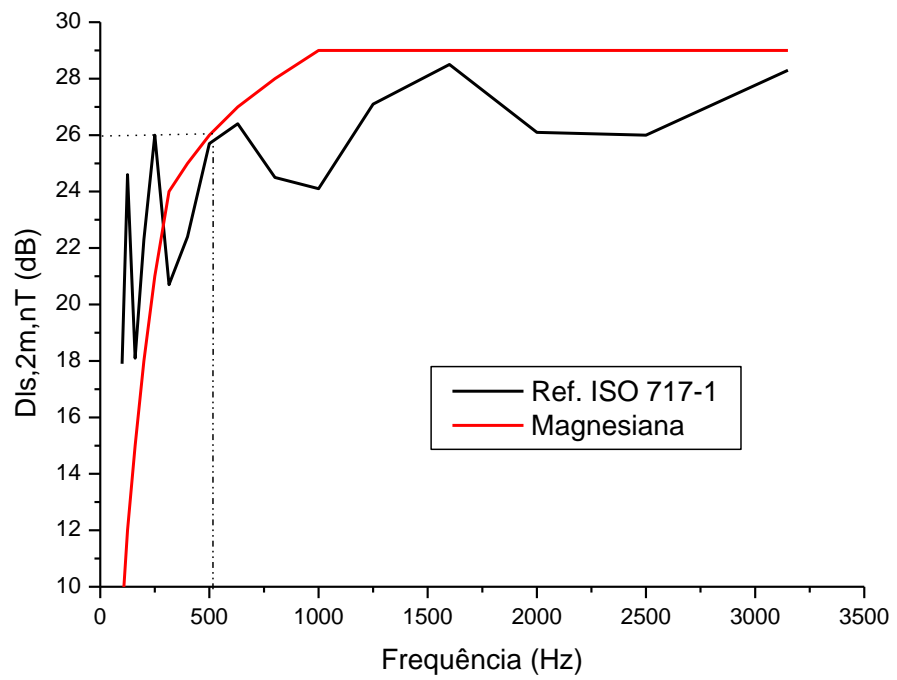

Figura 8: Curvas da Diferença Padronizada de Nível ajustadas com Curva de referência da norma ISO 717-1 para a fachada Sorel.

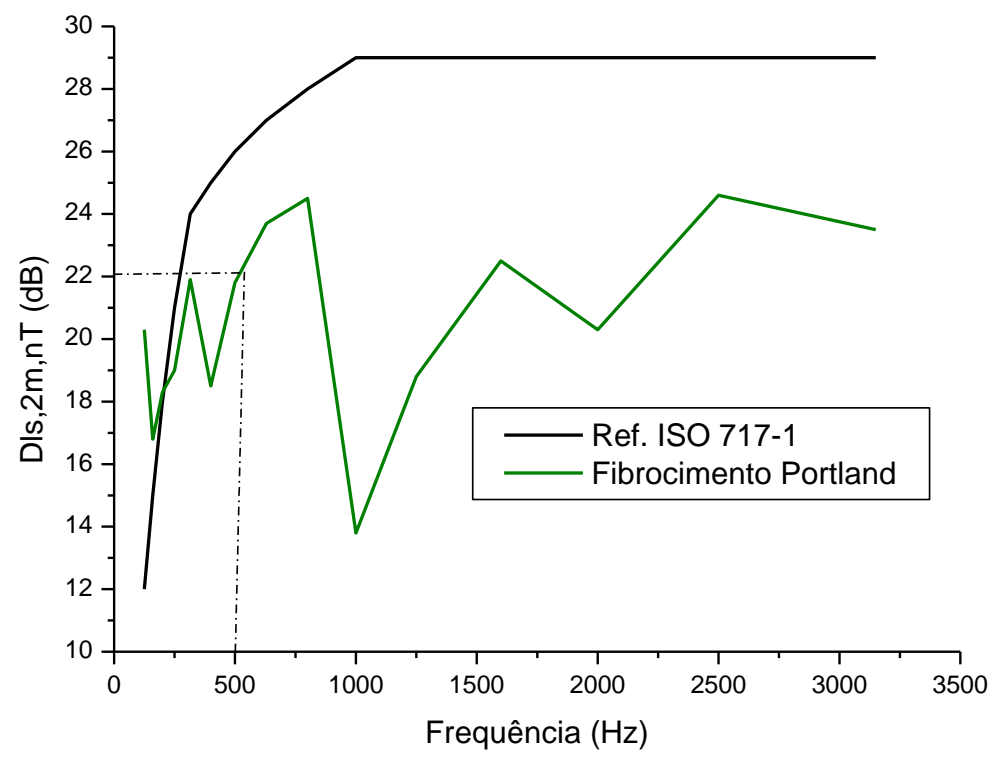

Figura 9: Curvas da Diferença Padronizada de Nível ajustadas com Curva de referência da norma ISO 717-1 para a fachada Portland.

Os valores de D2m,nT,w obtidos para as placas de fibrocimento Portland e magnesiana com a aplicação do procedimento da ISO 717-1[5] são apresentados na Tabela 5.

Tabela 5: Diferença Padronizada de Nível Ponderada.

\begin{tabular}{c|c}
\hline Tipo de cimento & $\mathrm{D}_{2 \mathrm{~m}, \mathrm{nT}, \mathrm{w}}(\mathrm{dB})$ \\
\hline Placa de Fibrocimento & 22 \\
\hline Placa magnesiana & 26 \\
\hline
\end{tabular}


Observa-se uma elevação de $4 \mathrm{~dB}$ no comportamento de isolamento das placas devido a substituição do cimento Portland pelo cimento com oxido de magnésio. Em questão de percepção, essa diferença de 4dB corresponde a uma mudança entre perceptível e notável. Por fim, considerando os critérios de isolamento de fachadas da norma NBR 15575-4 [8], esse resultado indica a mudança de classificação de desempenho mínimo para intermediário na classe de ruído I.

\section{CONCLUSÕES}

O presente trabalho buscou analisar sob a ótica do desempenho acústico um elemento construtivo alternativo à construção civil brasileira. As placas planas magnesianas podem ser entendidas como uma nova proposta para sistemas construtivos a seco, em especial, em substituição as placas tradicionais à base de fibrocimento.

Destaca-se que a melhoria do isolamento sonoro de sistemas de fachadas é progressivamente mais requerida, ficando esta preocupação mais acentuada com norma de desempenho NBR 15575 [8], que tem estabelecido critérios mínimos de isolamento sonoro aéreo de fachadas de edificações habitacionais.

Os resultados do isolamento sonoro aéreo mensurado pelo parâmetro acústico Diferença Padronizada de Nível em função de frequência mostraram um incremento no isolamento na maior parte das faixas de frequência para a placa que empregou o oxido de magnésio. Houve uma melhora significativa no isolamento sonoro aéreo na frequência de $1000 \mathrm{~Hz}$ onde foi possível excluir o efeito de coincidência que reduzia o isolamento nessa banda de frequência. Em termos globais, o parâmetro D2m,nT,w é indicado para comparação de desempenho acústico de isolamento entre sistemas construtivos. Nesse estudo verificou-se um aumento de $4 \mathrm{~dB}$ na substituição da placa de fibrocimento convencional (Portland) pela placa à base de óxido de magnésio. Isso representa uma mudança no atendimento da norma NBR 15575-4 [8], passando o isolamento sonoro aéreo da fachada do nível de desempenho mínimo para um nível intermediário, constituindo, assim, uma alternativa viável na execução de fachadas a partir da construção a seco.

\section{AGRADECIMENTOS}

Os autores agradecem à TESSA Engenharia, pela doação de todos os materiais de pesquisa, e, também, ao LACAF/Unicamp, pelos equipamentos utilizados. Agradecem também às instituições fomentadoras de pesquisa CAPES e CNPQ.

\section{BIBLIOGRAFIA}

[1] CNI. Confederação Nacional da Indústria. Indústria brasileira de cimento: Base para a construção do desenvolvimento. Associação Brasileira de Cimento Portland. - Brasília : CNI, 2012.

[2] HUMPHREYS, K., MAHA MAHASENAN, M., "Toward a Sustainable Cement Industry", 2002, http://www.wbcsdcement.org/pdf/battelle/sub_co2.pdf. Acesssado em Setembro de 2017.

[3] THOMAS, R. MgO Board., "Walls and Ceilings", 2007. Disponível em: <http://www.wconline.com/articles/85449-mgo-board?>. Acesso em 30/03/2017.

[4] BEIJING REPORT, Independent Environmental Assessment: Beijing 2008 Olympic Games, Published by the United Nations Environment Programme (UNEP) in February 2009.

[5] TAMBOLI, A., LEONARD, J. UMKANT, V., et al., CTBUH 8th World Congress, Dubai, March 3-5, 2008.

[6] SHAND, M. A., The Chemistry and Technology of Magnesiam. John Wiley \& Sons, Ltd., Hoboken, 2016.

[7] WORLD HEALTH ORGANIZATION (WHO), Guidelines for community noise, http://www.who.int/docstore/peh/noise/Comnoise3.htm. Acessado em Setembro, 2017.

[8] ASSOCIAÇÃO BRASILEIRA DE NORMAS TÉCNICAS (ABNT). NBR - 15575 - 4: Requisitos para sistemas de vedações verticais internas e externas- Edificações habitacionais - Desempenho. Rio de Janeiro, 2013.

[9] INTERNATIONAL ORGANIZATION FOR STANDARDIZATION. "ISO 717-1: Acoustics - Rating of sound insulation in buildings and of buildings elements - Airborne sound insulation", 2013.

[10] INTERNATIONAL ORGANIZATION FOR STANDARDIZATION. "ISO 140-5: Acoustics - 
Measurements of sound insulation in buildings and of buildings elements - Field measurements of airborne sound insulation of facade elements and facades." Geneva.1998.

\section{ORCID}

Thais Sacomani Zenerato

https://orcid.org/0000-0003-3018-4640

Stelamaris Rolla Bertoli

https://orcid.org/0000-0001-6140-6117

Carlos Eduardo Marmorato Gomes

https://orcid.org/0000-0002-4366-6027

Juliana Furtado Arrobas Martins

https://orcid.org/0000-0002-7255-5561

Patricia Stella Pucharelli Fontanini

https://orcid.org/0000-0002-7532-7505 